\title{
Liderazgo desde la perspectiva de la Red de Internacionalización Educativa Policial
}

\author{
Leadership from the perspective of the Police Educational Internationalization \\ Network
}

\section{Liderança a partir da perspectiva da Rede de Internacionalização Educacional Policial}

\author{
Alba Luz Molina-Garzón ${ }^{a *}$ | Jonatan Moreno-Dazab | Daysi Velásquez-Aponte \\ a https://orcid.org/0000-0002-4259-2986 \\ ${ }^{\mathrm{b}}$ https://orcid.org/0000-000 I-6903-4047 \\ c https://orcid.org/0000-0002-6790-4798 \\ Policía Nacional de Colombia, Bogotá, Colombia
}

- Fecha de recepción: 2020-03-30

- Fecha concepto de evaluación: 2020-06-18

- Fecha de aprobación: 2020-07-27 https://doi.org//0.22335/rlct.v| 2i3. I I86
Para citar este artículo / To reference this article / Para citar este artigo: MolinaGarzón,A. L., Moreno-Daza, J., \& Velásquez-Aponte, D. (2020). Liderazgo desde la perspectiva de la Red de Internacionalización Educativa Policial. Revista Logos Ciencia \& Tecnología, I2(3), 84-97. https://doi.org/I0.22335/rlct.v12i3.II 86

\section{RESUMEN}

El presente artículo compendia aspectos fundamentales para la Red de Internacionalización Educativa Policial Rinep -órgano colegiado entre sistemas de educación policial y homólogos en seguridad-, toda vez que investigadores de diversos países se unieron en el propósito de diseñar una propuesta formativa en liderazgo policial a partir del análisis de las particularidades y estilos, así como de la identificación de las diferencias y las similitudes en el ejercicio del liderazgo, como un aporte a las estrategias de cooperación e integración de cuerpos de policía miembros de la red. Metodológicamente el estudio se circunscribe en un enfoque descriptivo con un análisis cualitativo y un proceso de triangulación hermenéutica para la definición de categorías y análisis de la información. Por medio de los resultados obtenidos se establecieron tipologías en el ejercicio del liderazgo, comprensiones y conceptualizaciones disímiles, así como la prioridad de procesos formativos en liderazgo policial; circunstancias que hicieron denotar la necesidad de consolidar una propuesta cimentada en el ejercicio investigativo y las expectativas académicas, sustentada en el Diplomado Internacional en Liderazgo Policial como un escenario que coadyuve a la profesionalización del servicio de policía en beneficio de la seguridad y convivencia ciudadana en cada país.

Palabras clave: cooperación, formación, integración, liderazgo, policía 


\begin{abstract}
This article summarizes fundamental aspects for the Police Educational Internationalization Network (RINEP) - a collegiate body that connects the police educational systems and their security counterparts - given that researchers from different countries joined together with the purpose of designing a training proposal in police leadership based on the analysis of particularities and styles, as well as the identification of differences and similarities in the exercise of leadership, as a contribution to the cooperation and integration strategies of police force members in the network. Methodologically, the study is circumscribed in a descriptive approach with a qualitative analysis and a hermeneutical triangulation process for the definition of categories and information analysis. Through the results obtained typologies were established in regards to the exercise of leadership, understandings and dissimilar conceptualizations, as well as the priority of police leadership training processes; circumstances that denoted the need to consolidate a proposal based on investigative exercise and academic expectations, based on the International Diploma in Police Leadership as a scenario that contributes to the professionalization of the police service for the benefit of security and citizen coexistence in each country.
\end{abstract}

Keywords: cooperation, training, integration, leadership, police

\title{
RESUMO
}

O presente artigo busca sintetizar aspectos fundamentais para a Rede de Internacionalização Educativa Policial Rinep —órgão colegiado entre sistemas de educação policial e homólogos em segurança—, uma vez que pesquisadores de diversos países se uniram no propósito de desenhar uma proposta formativa em liderança policial partindo da análise das particularidades e estilos, assim como também da identificação das diferenças e similitudes no exercício da liderança, como uma contribuição às estratégias de cooperação e integração de corpos de Polícia membros da rede. Metodologicamente, o estudo se circunscreve em uma abordagem descritiva com una análise qualitativa e um processo de triangulação hermenêutica para a definição de categorias e análise da informação. Por meio dos resultados obtidos, se estabelecerão tipologias no exercício da liderança, compreensões e conceitos diferentes, bem como a prioridade de processos formativos em liderança policial; circunstâncias que fizeram denotar a necessidade de consolidar uma proposta fundamentada no exercício da pesquisa e as expectativas acadêmicas, sustentada no Diplomado Internacional em Liderança Policial como um cenário coadjuvante com a profissionalização do serviço de polícia em benefício da segurança e convivência cidadã em cada país.

Palavras-chave: cooperação, formação, integração, liderança, Polícia

La Red de Internacionalización Educativa Policial (Rinep) se creó en el 2016 y la integran 38 instituciones miembro: 30 Cuerpos de Policía, tres organismos multilaterales y cinco instituciones de educación superior, entre ellas la Dirección Nacional de Escuelas (Dinae) perteneciente a la Policía Nacional de Colombia. El direccionamiento estratégico de la red se orientad a trabajar las siguientes líneas de acción: (a) internacionalización curricular, (b) investigación, (c) movilidad académica, y (d) acreditación internacional. Estas líneas son los pilares fundamentales de esta comunidad académica que busca profesionalizar el servicio de policía en pro de la seguridad y convivencia ciudadana en cada nación.
A partir de esta lógica, la línea de acción "Internacionalización de la investigación” cobra importancia toda vez que se ha constituido en una de las grandes apuestas en el marco de la Rinep, con el propósito de otorgarle un mayor aprovechamiento a la generación de saberes de manera conjunta desde la formulación y ejecución de un proyecto de investigación, orientado a propiciar escenarios de reflexión, discusión y análisis, como aporte a las estrategias de cooperación e integración de cuerpos de policía miembros de la red.

En este sentido, ante la presencia de una sociedad cambiante y globalizada, las instituciones policiales se ven abocadas a reflexionar sobre el contexto pedagógico, las 
metodologías y las competencias que le otorgan a los policías para hacer frente a la realidad actual, así como un nuevo enfoque en la educación dirigido a optimizar un ejercicio del servicio policial coherente y consecuente con las necesidades reales de la comunidad, y conducido por buenas prácticas profesionales y humanas en el cumplimiento de su labor (Pedraza, Villamizar \& Ortiz, 2015).

Por tanto, la investigación se constituyó en una ventana de oportunidades para los investigadores, los cuerpos de policía participantes y los demás miembros vinculados a la Rinep, quienes se beneficiarán a partir del reconocimiento de las particularidades y las tipologías de liderazgo de instituciones policiales en una perspectiva comparada sobre sus sinergias, la diversidad de perfiles y expectativas, por cuanto se focalizó en cómo los líderes actúan y se relacionan entre sí.

Cabe señalar que el propósito de desarrollar un tema sobre liderazgo policial permitió que los investigadores se constituyeran en protagonistas y partícipes de nuevas interacciones, en las cuales se tuvo la posibilidad de contribuir de manera sistemática y continuada al fortalecimiento del desarrollo profesional de las instituciones policiales, así como compartir buenas prácticas, favorecer la cooperación internacional, promover el intercambio de experiencias y conocimiento, de oportunidades de aprendizaje y el mejoramiento continuo. Todos estos elementos que caracterizan a la sociedad actual, de conformidad con lo determinado por la Organización para la Cooperación y el Desarrollo Económicos (OCDE, 2008) y la Organización de las Naciones Unidas para la Educación, la Ciencia y la Cultura (Unesco, 1998).

De esta manera, los objetivos formulados se orientaron a plantear una propuesta formativa en liderazgo policial mediante el análisis estructural de las particularidades y estilos, así como la identificación de las diferencias y similitudes a partir del ejercicio del liderazgo como aporte a las estrategias de cooperación e integración de cuerpos de policía miembros de la Rinep.

La ruta metodológica se enmarcó en un estudio que se circunscribe en el enfoque descriptivo con un análisis cualitativo de contenido, conducente a un proceso de triangulación hermenéutica de acuerdo con lo propuesto por Cisterna (2005), de modo que fue posible establecer las categorías para facilitar el trabajo y el tratamiento de los datos.
La consecución de la información se realizó mediante el diseño tanto de las técnicas como de los instrumentos, los cuales se validaron por medio de videoconferencias concebidas como el escenario virtual colaborativo propicio para la generación de propuestas, recomendaciones y reflexiones en torno al tema objeto de estudio. Así, en este escenario - atendiendo la convocatoria- se hicieron presentes nueve países, 14 cuerpos de policía e instituciones de educación superior, representados por 35 investigadores designados por cada institución.

Los resultados obtenidos permitieron consolidar una propuesta de carácter pedagógico fundamentada en el ejercicio investigativo, las expectativas académicas y los intereses propios de cada país. Esta propuesta se sustenta en el Diplomado Internacional en Liderazgo Policial.

\section{Consideraciones teóricas}

El liderazgo como concepto se ha abordado desde diversas disciplinas y teorías a lo largo de los años. La academia, las empresas y los Estados se han interesado por definir qué es el liderazgo, qué identifica a un líder y cuáles son las diferentes características y modelos.

Cabe señalar que la dificultad de definir el liderazgo radica en que, según Bass (1990), existen tantas definiciones del concepto como personas intentando definirlo (como se cita en Sánchez 2010); por otra parte, de acuerdo con Northouse, el buen liderazgo significa diferentes cosas para diferentes personas (2013, como se cita en Flynn \& Herrington, 20I5).

En la literatura es posible encontrar una gran variedad de definiciones, entre estas la que proporcionan Avolio y Bass (2004), quienes al dirigirse a la base del concepto especifican liderar como "influir a las personas a conseguir algún objetivo particular definido". De acuerdo con Yukl y Van Fleet (1992), existen autores que describen el liderazgo como un proceso de influencia, en el que coinciden aspectos relevantes para analizarlo y explicarlo, entre otros, los rasgos particulares, las pautas de interacción, el desempeño de funciones, la percepción de quienes lo siguen, la influencia en las metas e intervenir en la cultura organizacional.

Por otra parte, otros autores defienden que dirigir y liderar son conceptos distintos y los plantean como afines. 
Entre los que diferencian los significados se encuentran Bennis y Nanus (1985), quienes proponen que "los directores son personas que hacen cosas bien (things right) y los líderes son personas que hacen lo correcto (right thing)". De igual manera, Zaleznik (1977) planteó que "los directores se ocupan de cosas que deben ser hechas $y$ los líderes de las cosas que importan a las personas".

A su vez, Yukl y Falbe (1991) diferencian las fuentes de poder derivadas de la posición (poder, autoridad, control) de las derivadas de la persona (capacidades, experiencia, competencias), de manera que permiten diferenciar a un director de un líder.

En este sentido, Espinoza-Parra, Molero y Fuster-Ruiz de Apodaca (2015) recalcan que, en el contexto del entrenamiento militar, el liderazgo transformacional aumenta el valor intrínseco de los esfuerzos realizados por los seguidores y las metas compartidas por los miembros del grupo. Prueba de esto es el hecho de que al hacer que sus seguidores vean sus esfuerzos como sacrificios cargados de significado personal, este tipo de líder logra transformar los objetivos personales en una visión compartida que da expresión a una identidad colectiva.

Dentro de este contexto, también el liderazgo policial ha sido objeto de estudio en el campo académico desde 1996 con Bryman, Kerr y Jermier. Las investigaciones al respecto están enfocadas, en su mayoría, en lo individual, esto es, en características y comportamientos (Hoggett, Redford, Toher \&White, 2018).

A nivel global los departamentos de policía buscan herramientas para favorecer su funcionamiento, ser más competitivos y fortalecerse institucionalmente. Según Salas (2016) y Toledo (20I4), como se citan en Nieto, Nieto y Moreno (2018), para proyectar instituciones policiales en un mundo en constante evolución es importante contar con líderes que contribuyan a la creación e implementación de estrategias de adaptación. Entonces, si se desea comprender por qué el liderazgo es relevante para instituciones como la policía, es pertinente definir el concepto, identificar sus características, indicadores y modelos, a la vez que cómo este concepto se aplica en diferentes áreas del mundo.

Day (20II, como se cita en Flynn y Herrington, 20I5) señala que es importante distinguir entre entrenar líderes y desarrollarlos; el primer caso hace referencia a que existen ciertos conocimientos que un líder debe tener respecto a problemas identificados; el segundo se refiere a crear en las personas la capacidad de responder a lo imprevisto. Los policías se ven constantemente enfrentados a retos que ponen a prueba sus capacidades personales y profesionales, pues es común que en varios días ellos no sepan qué actividad deban desarrollar o qué desafío deban superar. Es pertinente aceptar que el liderazgo es una palabra cuya definición es compleja, y las labores de policía también lo son; se desarrollan en un ambiente con múltiples elementos móviles que operan como sistema y requieren alta coordinación frente a múltiples situaciones o amenazas (Flynn \& Herrington, 20I5).

En ese sentido, para la Oficina de Naciones Unidas contra la Droga y el Delito (UNODC, 20I I), existe una estrecha relación entre el liderazgo, la cultura y la conducta. El liderazgo policial puede y debe garantizar un sistema disciplinario efectivo tanto a nivel interno (de la institución) como externo (con los civiles u otras instituciones). Eso significa que un miembro de la policía no solo debe comportarse de cierta manera, sino que también su comportamiento contagia a sus pares, lo cual resulta en una mejor convivencia entre ellos y se refleja también fuera de la institución.

En el caso colombiano, la Policía Nacional establece que las nuevas realidades exigen un acercamiento institucional a través del Modelo Holístico de Liderazgo Policial (MHLP). Desde el punto etimológico, lo holístico sugiere que "un todo no se explica por las partes que lo componen por sí solas, si no que ese todo determina cómo se comportan las partes" (Nieto et al., 2018). Este modelo aborda múltiples asuntos: los objetivos, los entornos, los comportamientos, la diferencia entre mando dirección y liderazgo, los espacios, los atributos del policía líder, los estilos de liderazgo y los alcances, entre otros.

Para Nieto et al. (2018), algunos de los comportamientos asociados al liderazgo son la capacidad de un individuo de establecer objetivos claros y ambiciosos en estrategias novedosas; planificar y asignar tareas de acuerdo con las competencias de las personas; motivar a los miembros del equipo y reconocer sus logros; además de ejercer la autoridad de forma efectiva. Todos estos comportamientos son aplicables a la labor policial.

De acuerdo con la Asociación Internacional de Jefes de Policía (IACP) (2006, como se cita en Nieto et al., 20I8), se define el liderazgo como un proceso capaz de incidir en el comportamiento humano con el fin de alcanzar las 
metas de la organización que sirven al público y, al mismo tiempo, desarrollar personas, grupos y organizaciones.

Cabe señalar que la comunidad, dentro de sus expectativas, espera que el policía no se dedique solo a identificar, diagnosticar y plantearle alternativas para darle solución a las dificultades que se le presenten, sino que considera fundamental también que la debe orientar mediante campañas y jornadas educativas, de manera que la comunidad aprenda a organizarse, a definir y priorizar necesidades y problemas, al igual que las posibles soluciones relacionadas con la seguridad y la convivencia; asimismo, a cómo elaborar planes individuales y grupales en los términos de una acción ciudadana permanente (Paz, 2009).

Ahora bien, en el marco de las instituciones adscritas a la Rinep se encontraron algunos documentos relacionados con la necesidad de dar claridad a los elementos de la función policial, a la definición específica, en términos doctrinales de mando y liderazgo, y a las características del estilo de liderazgo que tienen los policiales. Entre otros escritos, se señalan los siguientes: "Manual de don de mando y liderazgo” (República Dominicana),“Manual de instrucción básica don de mando y liderazgo policial” (Honduras), "Cuaderno de trabajo: valores éticos y jurídicos del curso de formación inicial-policía estatal acreditable" (México). Así, se sustenta el propio interés de la investigación propuesta para la red, toda vez que la multiplicidad de términos, estilos y enfoques que pululan sobre el término liderazgo urgen de una respuesta que marque la diferencia para los miembros de esta red, fortaleciendo sus vínculos y las líneas de acción sobre las cuales cada cuerpo policial aporta para la consolidación de proyectos perdurables en el tiempo.

Se subraya, además, la equivalencia de los términos en las definiciones preponderantes de las investigaciones sobre el tema de liderazgo, en las cuales se evidencia un margen poco claro tanto en las concepciones como en las teorías que los sustentan y en la dificultad para disgregar estos términos que se establecen como similares. Por otra parte, la descripción escueta de "elementos" atañe a las características que la investigación recoge y sintetiza como los elementos que fundamentan el quehacer policial, sin hacer una aportación especial al liderazgo como característica que pudiera entenderse como particular para este cuerpo policial.

\section{Metodología}

Esta investigación se enmarcó bajo un enfoque descriptivo con un análisis cualitativo, toda vez que proporcionó elementos para interpretar y actuar frente a determinadas situaciones y contextos (Hernández, Fernández \& Baptista, 2014); conducente, además, a un proceso de triangulación hermenéutica, de acuerdo con lo propuesto por Cisterna (2005), de modo que fue posible establecer las categorías para facilitar el trabajo y el tratamiento de los datos.

De acuerdo con Sartori (como se cita en Goodrick, 2014), fue necesario realizar un estudio comparativo, lo que significó observar similitudes y diferencias; con este método se trató de explicar y analizar para comprender los elementos propios de, al menos, dos estamentos. Asimismo, el mismo Goodrick (2014) explicita que "aplicar la comparación implica que dos fenómenos u objetos de estudio posean propiedades en común que permitan identificar atributos o dimensiones parcial o totalmente similares o disímiles. Así, se podrán destacar sus peculiaridades".

Adicionalmente, esta metodología permitió evaluar si las teorías que fundamentaban un programa de acción o un programa de cambio podrían resultar efectivas. Para este caso, constituir los elementos que caracterizaban similitudes y diferencias entre los distintos cuerpos de policía en torno al tema del liderazgo policial permitió establecer una línea de base para hacer propuestas que aportaran a las estrategias de cooperación e integración de cuerpos de policía de la Rinep.

Una de las estrategias seleccionadas para alcanzar los objetivos propuestos del proyecto fue los encuentros virtuales como alternativa de interacción con los miembros de la Rinep, con lo cual se logró generar una mayor flexibilidad en el intercambio de saberes y experiencias, superando las barreras de la distancia mediante la comunicación simultánea en tiempo real. En este contexto, la obtención de la información fue posible mediante el diseño y la validación de las técnicas (encuesta y grupo focal) y los instrumentos (cuestionario y guion de entrevista grupal) por medio de este escenario virtual colaborativo adecuado para la generación de planteamientos, recomendaciones y reflexiones en torno al objeto de estudio. Se constituyó, entonces, un equipo de investigadores que representaban nueve países, 14 cuerpos de policía e 
instituciones de educación superior, representados, a su vez, en 35 profesionales entre policiales y docentes.

A discreción de cada institución, la transcripción de la discusión favoreció la identidad de los informantes, quienes en su mayoría fueron codificados para no sesgar la información revelando sus identidades. En este orden de ideas, se homogeneizaron los códigos de los informantes para el proceso de análisis de modo que fuera posible filtrar las respuestas $y$, de esa manera, encontrar una amplitud en la información sobre el tema investigado.

Una vez recopilada la información, se procedió a realizar un análisis cualitativo de los datos mediante la técnica de análisis cualitativo de contenido propuesta por Bardin (2002). Esta técnica facilitó en la etapa de preanálisis organizar, clasificar, codificar la información y crear unidades de análisis. De este modo, se establecieron las categorías que permitieron facilitar el trabajo y el tratamiento de los datos, conducente a un proceso de triangulación cualitativa de acuerdo con la propuesta de Cisterna (2005).

La triangulación hermenéutica se hizo necesaria en esta investigación toda vez que permitió cuatro tipos de cruzamientos:

- Triangulación por cada estamento. Implicó la comparación inferencial de la opinión sobre un tema de una población con un tópico de investigación.

- Triangulación de información entre estamentos. Tuvo un carácter interestamental y permitió hacer comparación entre sujetos indagados.

- Triangulación entre diversas fuentes de información. Propicia para las investigaciones que usan más de un instrumento de recopilación de datos, ya que permite recopilar información por estamentos y realizar el cruce dialéctico de la información pertinente al objeto de estudio; en este caso, la obtenida en el trabajo de campo (cuestionario y entrevista grupal), los resultados del proceso de comparación.

- Entrecruzamiento con la literatura existente sobre liderazgo policial. Para la construcción de la propuesta estratégica.

\section{Resultados}

Los resultados obtenidos permitieron consolidar una propuesta de carácter formativo cimentada en el ejercicio investigativo, las expectativas académicas y los intereses propios de cada país miembro. Se iniciará con la caracterización de las particularidades y los estilos de liderazgo que identifican los cuerpos de policía vinculados a la Rinep.
En primera instancia, se reconoce que existen, a nivel institucional, programas para la formación del profesional de policía, para el ascenso en la carrera y el mejoramiento constante de su función policial. Sin embargo, como tal no se mencionan cátedras especializadas sobre el tema, los cursos de ascenso no señalan en qué modalidad son y tampoco su duración o nivel de formación o educación continuada.

Este primer punto de la encuesta permitió visibilizar que cada uno de estos países tiene en sus propias particularidades una concepción que, a su vez, converge en varios aspectos de las teorías del liderazgo, mantiene sus propias postulaciones frente al mando y la dirección. Estas particularidades se contextualizan en la realidad institucional de cada informante. También revelan el papel del líder policial en cada país, de modo que de lo anterior se establecen de forma progresiva unas primeras características del liderazgo policial que aparecen como similitudes y diferencias entre los miembros de la Rinep.

De los estilos de liderazgo más reconocidos, los considerados por los informantes como los predominantes en su institución son: autocrático, democrático/participativo, situacional, jerárquico, burocrático y transformacional.

Esta categorización refleja, en primera instancia, un estilo de liderazgo dominante. Con estas particularidades iniciales se van presentando las diferencias dadas por la cultura de liderazgo que existe en el contexto local de cada cuerpo policial. En el ámbito policial los estilos de liderazgo los determina única y exclusivamente la situación. Los que tienen cargos de tareas y dirección, desarrollan mando y dirección y lo verbalizan como liderazgo, de manera que es esta confusión conceptual uno de los primeros problemas identificados.

Es necesario precisar aquí, entonces, que a estos estilos subyacen diversas teorías de liderazgo, entreverando elementos de la función del líder empresarial y los enfoques o estilos gerenciales de tipo estratégico y eficaz.

Se evidenció que el estilo burocrático se ubica en un nivel inferior, probablemente por el desgaste del concepto que, en los diversos contextos, se concibe como un estilo de liderazgo centrado en la sumisión y no en la gestión, lo que puede acercarse incluso más a las teorías tradicionales propias del estilo autócrata, orientado a la tarea (Reddin, 1983). 
Por su parte, el estilo democrático —el cual se presenta en las teorías siempre en contrastación con el autocrático y el liberal- no presenta una posición preponderante, entendiendo la toma de decisiones colectiva como un estilo de liderazgo eficiente y orientado al logro. Lewin, Lippitt y White (1939), al intentar conceptualizar los estilos de liderazgo, identificaron que el estilo democrático propicia un mejor comportamiento grupal.

Ahora bien, las teorías sobre liderazgo situacional mencionan la necesidad del reconocimiento del contexto para que la eficacia del liderazgo se considere con efecto a largo plazo. Hersey y Blanchard (1967) sostienen que la organización permite el crecimiento del liderazgo situacional desde una dimensión personal que depende de unas situaciones a partir de las cuales el sujeto va fortaleciendo sus habilidades de liderazgo. El éxito de este tipo de liderazgo se centra en la aceptación de los demás miembros de la comunidad. Aunque el estilo situacional es uno de los términos de mayor referencia, no es uno de los estilos predominantes en el interior de las organizaciones.

Según Bass y Avolio (1993) el estilo transformacional exige un proceso sobre la base de la motivación, desde el cual se responde a los intereses y las necesidades de los seguidores del líder, lo que favorece los grandes cambios en las organizaciones.

Finalmente, la más alta posición la tiene el estilo autocrático, el cual evidentemente responde a la estructura jerárquica de los entes policiales y se sustenta en diversas teorías en las que la autoridad es la base del logro de los procesos, del alcance de los objetivos y de la transformación institucional. En sus investigaciones, Lewin, Lippitt y White (1939) establecen que este estilo, si bien se encamina al logro de la tarea, también favorece comportamientos hostiles y agresivos, propios de la competitividad.

Esta diversidad de estilos confluye con la información relacionada en la Tabla I, la cual se obtuvo a partir de la realización de un diagnóstico sobre liderazgo policial, lo cual permitió identificar las características o las cualidades sobresalientes que a criterio de los investigadores debe tener un líder policial, entre las que se destacan las que se presentan en la tabla $\mathrm{I}$.

Ahora bien, en este análisis descriptivo es posible constatar también algunas características, cualidades o atributos comunes entre los países participantes, en lo que se con-
Tabla I. Características o cualidades del líder policial

\begin{tabular}{|c|c|c|c|}
\hline País I & Visionario & $\begin{array}{l}\text { Dirección } \\
\text { y delegación }\end{array}$ & Flexible \\
\hline País 2 & $\begin{array}{l}\text { Habilidades } \\
\text { comunicativas }\end{array}$ & $\begin{array}{l}\text { Inteligencia } \\
\text { emocional }\end{array}$ & Autoreconocimiento \\
\hline País 3 & Integridad & Disciplinado & Innovador \\
\hline País 4 & Actitud crítica & Argumentativo & $\begin{array}{l}\text { Respeto por } \\
\text { las diferencias }\end{array}$ \\
\hline País 5 & Honesto & Comprometido & Transformador \\
\hline País 6 & $\begin{array}{l}\text { Solucionador } \\
\text { de conflictos }\end{array}$ & Voz de mando & Empatía \\
\hline País 7 & $\begin{array}{l}\text { Pensamiento } \\
\text { estratégico }\end{array}$ & $\begin{array}{l}\text { Facilitador } \\
\text { de estrategias }\end{array}$ & Vision estratégica \\
\hline País 8 & Creativo & $\begin{array}{l}\text { Capacidad } \\
\text { de planificar }\end{array}$ & $\begin{array}{l}\text { Centrado en los } \\
\text { resultados }\end{array}$ \\
\hline País 9 & $\begin{array}{l}\text { Objetivos y } \\
\text { metas claras }\end{array}$ & Solidario & Vocación de servicio \\
\hline
\end{tabular}

sidera debe tener un líder policial. Entre otras, se encuentran visión estratégica, solidaridad, disciplina, inteligencia emocional, innovación y las habilidades de comunicación. Se entiende, entonces, que esta clasificación de las características se ha adelantado desde la mirada particular de algunos miembros de cada cuerpo policial vinculado en el estudio comparativo, desde quienes estas características en las que se converge plantean unos intereses comunes para establecer la línea base que da sustento a la propuesta integradora con la cual cristalizar la misión de la Rinep.

Estas características están asociadas, más que a las teorías subyacentes a los estilos de liderazgo señalados como predominantes, a unos lugares comunes, propios del discurso que sobre liderazgo se maneja en el lenguaje de cada institución policial.

Los instrumentos diligenciados hacen referencia permanente a los conceptos básicos de liderazgo de modo genérico $y$, algunas veces, difuso, dada las múltiples acepciones del concepto que, seguramente, se estudian en los cursos sobre el tema de liderazgo que ofrecen los cuerpos de policía.

Ante la pregunta sobre programas específicos para capacitar y entrenar sobre liderazgo policial, como tal no se mencionan cátedras especializadas en el tema; los cursos de ascenso se presentan con escasas características sobre las modalidades, la duración o los niveles de formación a los que atañan: "Es una materia muy básica en los temarios de estudio para ascenso desde cabo segundo hasta 
capitán, no es un programa continuo, solo estudio para ascenso" (ILII)."Hay una propuesta para un proyecto de la Academia Nacional de Policía que está compuesto por: (I) curso de liderazgo en formación policial; (2) curso a distancia dirigido a todos los jefes; (3) cursos presenciales dirigidos a la alta dirección" (ILI5). "Desde la formación básica que es la inicial con los cadetes, la formación de reacreditación con policías y las capacitaciones anuales" (ILI7).

Estas voces que se subrayan como significativas, reflejan el interés institucional por darle relevancia al lugar que ha de ocupar la formación sobre liderazgo policial en el interior de las instituciones.

Se verá en la propuesta cómo los resultados de la investigación constatan la necesidad de relacionar un mínimo en el caso del liderazgo policial que identifique $o$ abarque a todos los miembros de la Rinep. Si bien varios estudios de los señalados dan cuenta de la emergencia de conceptos y teorías sobre liderazgo, en cada país se van dando algunos pasos para considerar el liderazgo policial como una potente oportunidad de profesionalizar el quehacer policial.

De los países que reportan tener algún proceso formativo singular se han tomado en cuenta los elementos constitutivos para considerarlos en el propósito de la investigación, a fin de que sean integrados en una propuesta que, teniendo en cuenta las realidades contextuales y resaltando estos avances en materia de formación sobre liderazgo fortalezcan los propósitos misionales de la red.

Para consolidar este primer bloque de particularidades, el instrumento de diagnóstico se propuso identificar los parámetros o lineamientos en los que debería estar fundamentado el liderazgo policial, a saber:

- visión estratégica;

- capacidad de dirección;

- dominio e inteligencia emocional;

- confianza en la toma de decisiones de su equipo;

- compromiso en la gestión;

- ser, saber y saber hacer;

- observancia de la doctrina institucional;

- indicadores de eficiencia, eficacia y efectividad;

- objetividad;

- promover el desarrollo humano;

- integración de los equipos de trabajo;

- asertividad en la comunicación;

- aplicabilidad de reglamentos.
Se puede observar que, al señalar parámetros o lineamientos sobre los que se debe fundamentar el liderazgo policial, se constatan varias divergencias reseñadas en la revisión de documentos institucionales, entre los cuales vale la pena destacar que se ponen en el mismo nivel conceptual aspectos tales como competencias (saber, saber hacer y ser), normas, características del liderazgo, parámetros y lineamientos, sin tener discreción sobre las distinciones ni precisar cómo estas se articulan con los estilos predominantes.

De manera subsiguiente, se describen las diferencias $y$ similitudes del liderazgo policial, información obtenida a partir de los grupos focales; se inicia con las similitudes.

En relación con las conceptualizaciones sobre liderazgo $y$ en virtud de las múltiples diferencias que existen en la teoría, se identifican estas mismas variaciones, dada la diversidad de entornos culturales que circundan cada cuerpo policial participante en la investigación y en atención a esta diversidad de contextos es que "amerita la estandarización de procesos formativos" (ILI4), y "se podrían implementar cátedras comunes” (ILI9).

A su vez, los instrumentos revelan que los miembros participantes en común tienden a considerar el liderazgo como una facultad innata que se cualifica mediante el ejercicio de la autoridad en condiciones de la función pública que se ejerce. Se lee, entonces, el liderazgo como facultad de una persona sobre otra, "en virtud de la superioridad jerárquica que ostenta y acorde a las funciones específicas que cumple, de disponer sobre sus subalternos ciertas condiciones atinentes al servicio policial” (ILII).

Del mismo modo, se concibe que "es la autoridad o facultad que ostentan algunos miembros de los diversos cuerpos de policía para tomar decisiones y emitir órdenes, con el fin de cumplir las funciones o tareas institucionales que se les hayan encomendado" (ILI2).Así como "es la autoridad o el poder que ejerce la persona que dirige respecto del personal subalterno o colaborador para tomar decisiones y hacer que las mismas se cumplan" (ILI3).

Tanto en los grupos focales como en el instrumento de liderazgo aparece el estilo de liderazgo participativo-democrático como uno de los más relevantes, sustentado en concepciones en las que coinciden las voces de los informantes: 
Líder es aquel jefe que se gana la voluntad de sus subalternos y obtiene su colaboración en forma voluntaria y consciente para lograr las reformas necesarias dentro de su unidad, persiguiendo siempre las mismas metas, objetivos $y$ compartiendo una visión (ILI7).

Asimismo,"es el arte de saber dirigir e influir en el comportamiento de las personas colaboradoras, de tal manera que se obtenga la confianza, respeto y cooperación voluntaria, para cumplir con éxito los objetivos institucionales" (ILI9). El liderazgo dentro de las características comunes,

[es] entendido tanto por las competencias particulares del líder, como por el reconocimiento como tal por parte de sus seguidores, constituyen los lados de un único fenómeno social, que se ha mencionado a lo largo de la historia y que ha evolucionado desde el punto de vista de las competencias personales del líder como del lado de la influencia, ejercida sobre sus seguidores en la lógica del reconocimiento de estos hacia su superior (ILIII).

Tras analizar sus voces, también se constata que el "don de mando" se considera en común como el "arte de imponer la voluntad sobre otros en forma tal de obtener obediencia, confianza, respeto y cooperación leal" (ILI4); se considera arte toda vez que se accede a la formación policial como medio para aprender a hacer cumplir los objetivos institucionales y en su generalidad (no exclusividad) está dirigida a los oficiales, quienes por autoridad del cargo pueden dar las órdenes que serán cumplidas por los agentes y tropas, cada nivel en su grado de responsabilidad en relación con la orden.

Este "don de mando" es unidireccional, verticalista y proviene del rango jerárquico obtenido dentro de la institución:"Se refiere al puesto de jefe de personal dentro de la Policía, es el elemento que ejerce el mando y disciplina en la unidad operativa que tenga a su cargo" (ILI5).

Se destaca, entonces, que en relación con la dirección, esta se considera "la capacidad de administrar los bienes y recursos humanos de la institución policial, siempre y cuando la prelación jerárquica lo ubique dentro de tal actividad" (ILI6).A su vez, se considera una forma de utilizar y distribuir los recursos de una institución policial para cumplir las funciones que le son encomendadas por las normas y los reglamentos nacionales.

Para sintetizar, se hallan similitudes en el interés de fortalecer y estandarizar la formación en el liderazgo policial dado que esto afianzará las conceptualizaciones y los factores relativos al "mando y dirección" en la carrera policial, centrados en las teorías y prácticas que generen motivaciones y acciones cada vez más profesionales en los distintos cuerpos policiales, propendiendo a transformar los estilos de liderazgo preponderantes. Así, se encuentran suficientes elementos para reconocer el liderazgo como un aspecto fundamental del quehacer policial que debe asentar diferencias entre las teorías administrativas y de gestión gerencial.

Estos elementos se identificaron como similares en el análisis y la triangulación mediante el cruce interestamental, lo cual permite identificar las categorías comunes, para este caso, coincidencias sobre los estilos de liderazgo y las concepciones sobre la dirección, el mando y el liderazgo que sustentan esta síntesis. Las matrices de análisis permitieron también la codificación que facilitó esta categorización, asignando porcentajes a la frecuencia con la que aparecen los términos o expresiones relacionadas directamente con cada categoría.

A continuación, se presentan las diferencias más relevantes que complementan a algunas de las expuestas en la primera parte de este apartado.

El término liderazgo puede estar relacionado o diluido en las conceptualizaciones de mando y dirección; he aquí unos ejemplos: "Capacidad para administrar a las personas y crear seguridad psicológica en el equipo" (ILI6). "Todo conductor policial debe impartir justicia en materia de ascensos y en la aplicación de castigos. Para ello se debe conocer la conducta humana y, saber por qué personas bajo las mismas circunstancias actúan de manera distinta" (ILI8). "Se puede definir como el poder de influencia que debe tener el superior sin violentar los derechos de los subalternos" (GFI00916). "El policía apoya a su gente, no es autoritario, está donde su gente lo necesita, los orienta y corrige" (GFI80915).

Por tanto, el liderazgo policial, si bien está enmarcado por los lineamientos de la doctrina policial, se ve entreverado por unas teorías que, al ser disímiles, generan procesos fallidos en los que la teoría es insuficiente, dado que existen diversos modos de comprensión de la teoría y el ejercicio del profesional de policía.

En la tabla 2 se compendian las diferencias y similitudes relacionadas con el liderazgo policial. 
Tabla 2. Diferencias y similitudes en el liderazgo policial

\begin{tabular}{|c|c|}
\hline Diferencias & Similitudes \\
\hline $\begin{array}{l}\text { Modos de comprensión y ejercicio } \\
\text { de la doctrina policial. }\end{array}$ & $\begin{array}{l}\text { Importante estandarizar el proceso } \\
\text { formativo de liderazgo. }\end{array}$ \\
\hline $\begin{array}{l}\text { Definiciones y teorías que circun- } \\
\text { dan los ejercicios de mando policial, } \\
\text { dirección o administración policial y } \\
\text { liderazgo policial. }\end{array}$ & $\begin{array}{l}\text { Falta reconocimiento del liderazgo } \\
\text { como elemento esencial del que- } \\
\text { hacer policial. }\end{array}$ \\
\hline $\begin{array}{l}\text { liderazgo policial. } \\
\text { Procesos formativos sobre liderazgo. }\end{array}$ & $\begin{array}{l}\text { Predominan los estilos de liderazgo } \\
\text { de teorías administrativas, al margen } \\
\text { del quehacer policial. }\end{array}$ \\
\hline \multirow{2}{*}{$\begin{array}{l}\text { Conceptualizaciones diversas sobre } \\
\text { liderazgo policial. }\end{array}$} & La toma de decisiones es vertical. \\
\hline & $\begin{array}{l}\text { No existen cátedras especializadas } \\
\text { sobre liderazgo policial. }\end{array}$ \\
\hline
\end{tabular}

Con todo y lo anterior, la investigación realizada como aporte a las estrategias de cooperación e integración de los cuerpos de policía miembros de la Rinep se promovió como una iniciativa que, de manera comparativa, permitiera reconocer entre las similitudes y las diferencias en torno al liderazgo policial, así como las posibilidades de actuación concreta y viable según los contextos propios de cada país, a la vez que se establecieran criterios orientadores y unificadores en torno al liderazgo policial, contribuyendo al cumplimiento de los objetivos estratégicos de la red.

En este sentido, la información obtenida a través de la aplicación de instrumentos y complementada con las teorías de trabajo reticular para la consolidación de apuestas transnacionales y los proyectos comunes, junto con el uso de las distintas redes tecnológicas, han de ser involucrados en diferentes niveles y con diversas estrategias para fortalecer la cooperación académica e investigativa. Los resultados de este estudio a nivel internacional así lo constatan, y las apuestas futuras incrementarán las posibilidades de que estos proyectos consoliden el quehacer investigativo en el interior de la Rinep.

Como soporte a esta iniciativa se pretende atender a la perspectiva más reconocida en el mundo moderno como la posibilidad de compartir experiencias, buenas prácticas y ejercicios exitosos que aporten a la consolidación de un saber propio del ser profesional de policía en cada contexto. Ese diálogo de experiencias compartidas busca ampliar los horizontes de posibilidades dado el abanico multicultural que atiende a la diversidad de culturas de los miembros de la red.

Por tanto, la propuesta planteada debe ser transversal a un proceso formativo que dé respuesta directa a la perspectiva de mayor interés denotado a partir de la investigación, como lo es la educación en liderazgo. Para conseguir este propósito se requirió de una estrategia que guiara a los cuerpos de policía que componen la Rinep; esta estrategia es el "liderazgo policial", en cuanto es un proceso interactivo y compartido, considerado un actor determinante en el propósito de establecer relaciones de cooperación e integración.

Es por esto que uno de los retos que se planteó en el marco de la investigación con los cuerpos de policía que conforman la Rinep estuvo orientado a definir las características y las tipologías en relación con el liderazgo policial, identificar en dónde convergen y cuáles pueden ser las nuevas perspectivas y el interés en el que se centra. De esta manera, se constituye en referente $y$ antecedente para la elaboración de una propuesta integradora que posibilite operacionalizar la misión de la red, compartiendo escenarios de construcción conjunta de saberes $y$, así, avanzar en la consolidación de un liderazgo policial por medio del cual transformar realidades a partir del quehacer policial.

Por esta razón surgió la idea de estructurar una propuesta formativa con el fin de fortalecer el liderazgo policial, consolidándose en el Diplomado Internacional en Liderazgo Policial (DILP), tal como se describe en las tablas 3, 4, 5 y 6 .

Tabla 3. Propósito del diplomado

Propósito del Diplomado Internacional en Liderazgo Policial

Operacionalizar la misión de la Red de Internacionalización Educativa Policial Rinep: "promover actividades de internacionalización educativa entre las instituciones de educación policial y homólogos en seguridad, generando proyectos de cooperación académica internacional e interinstitucional con el fin de permitir el aseguramiento de la calidad de estas organizaciones, aportando a la prospectiva de convivencia y seguridad ciudadana de cada país".

A su vez, proveer herramientas para la consolidación de una cultura de liderazgo policial en cada uno de los cuerpos policiales de la Rinep, fundamentando distintos niveles de actuación (personal-institucionalcomunitario); sustentado desde la teoría y su aplicación, y la comprensión del liderazgo policial en todas las instancias y dimensiones del ser integral.

Intensidad horaria: I 20 horas (semipresencial) Ciclos: I-II-III-IV

En esta propuesta curricular subyace la aplicación de las teorías en ejercicios prácticos que serán socializados mediante diversas plataformas tecnológicas para atender a varias de las perspectivas descritas, como, por ejemplo, el uso de redes, la construcción de conocimiento y compartir experiencias significativas para todos los miembros. La propuesta se enriquece con la participación de docentes

\section{.}


Tabla 4. Objetivos del diplomado

\section{Objetivos}

Impartir conocimientos teóricos y metodológicos que orientan el liderazgo policial, fomentando no solo el saber teórico, sino también el ejercicio práctico del policía en su función en el interior de la institución.

Desarrollar habilidades de liderazgo policial que permitan la aplicación concreta en situaciones reales en las que se reconozcan y analicen las necesidades y expectativas de la persona, la institución y la comunidad a la que le sirve.

Posibilitar la reflexión crítica sobre las competencias y habilidades que hacen viable un liderazgo policial, inspirado en la integralidad del ser al servicio de su profesión y vocación.

Tabla 5. Participantes en el diplomado

\section{Dirigido a}

Miembros de los distintos cuerpos policiales que integran la Rinep, particularmente a quienes tengan a su cargo responsabilidades de decisión y gestión, como aporte a la cualificación profesional.

Miembros de los cuerpos policiales, con la intención de mejorar su actuar como líder a partir de conocimientos profundos y prácticos en el tema de liderazgo policial aplicable en su contexto.

Instituciones adscritas a Rinep, comprometidas con la búsqueda de la efectividad como un concepto universal y transversal de la profesión policial y, por tal razón, relacionado y consecuente con el liderazgo policial.

internacionales, quienes harían sus aportes en cada curso o versión del diplomado internacional. Esta construcción colectiva de conocimiento favorecerá la promoción de prácticas de formación policial desde distintas perspectivas según el contexto internacional.

\section{Discusión}

A partir del ejercicio comparativo a través del cual se presentaron los resultados obtenidos fue posible evidenciar elementos que diferencian y asimilan el quehacer policial en las distintas latitudes a las que pertenecen las instituciones participantes y dan cuenta, a su vez, de las subjetividades propias de quienes en su ejercicio asumen el liderazgo policial como un elemento propio del servicio, en las que se entremezclan las teorías, los modos de actuar propios del contexto y aspectos de la personalidad de quienes sirven a la institución.

Los resultados confirman que el grado de confianza entre quien ejerce el liderazgo y quienes son liderados (Zanini, Migueles \& Colmerauer, 2014) está determinado por los estilos de liderazgo que predominan en la institución, mediados por los grados de motivación externa que
Tabla 6. Descripción general de contenido del diplomado

\begin{tabular}{|c|c|}
\hline Contenido & Descripción general \\
\hline $\begin{array}{l}\text { Módulo nivelatorio- } \\
\text { introductorio }\end{array}$ & $\begin{array}{l}\text { Cumple con el propósito de familiarizar a los } \\
\text { asistentes sobre los conceptos fundantes del } \\
\text { liderazgo policial, planteando las distinciones } \\
\text { relevantes sobre mando, dirección y liderazgo, } \\
\text { con el propósito de dar sustento teórico a la } \\
\text { propuesta de aplicación práctica del liderazgo } \\
\text { policial como una acción más allá de la teoría. }\end{array}$ \\
\hline $\begin{array}{l}\text { Ciclo I-Pensar y actuar } \\
\text { como líder policial }\end{array}$ & $\begin{array}{l}\text { Plantea el liderazgo policial como un proceso } \\
\text { mental en el que el líder se fortalece desde } \\
\text { su ser íntegro, contextualizado en un mar- } \\
\text { co situacional a partir del cual desarrolla un } \\
\text { diagnóstico para identificar los retos y las } \\
\text { oportunidades de mejora. }\end{array}$ \\
\hline $\begin{array}{l}\text { Ciclo II-Gestión } \\
\text { estratégica del } \\
\text { liderazgo policial }\end{array}$ & $\begin{array}{l}\text { Propone fijar el eje central en la visión } \\
\text { estratégica del cambio desde el liderazgo } \\
\text { policial, comprendiendo que cada institución } \\
\text { tiene su propio contexto cultural. Sobre una } \\
\text { línea base, esta gestión de cambio establece } \\
\text { unos mínimos fundantes que orienten la } \\
\text { acción de los sujetos que se forman como } \\
\text { líderes en el ejercicio policial. }\end{array}$ \\
\hline $\begin{array}{l}\text { Ciclo III-Proyección } \\
\text { integral del liderazgo } \\
\text { policial }\end{array}$ & $\begin{array}{l}\text { Plantea que la gestión de cambio se apoye en } \\
\text { los conceptos más relevantes estudiados a } \\
\text { nivel mundial y los proyecte a la acción inte- } \\
\text { gral del ser policía en comunidad, basándose } \\
\text { en procesos sociales. }\end{array}$ \\
\hline $\begin{array}{l}\text { Ciclo -Liderazgo } \\
\text { policial para la } \\
\text { mejora permanente }\end{array}$ & $\begin{array}{l}\text { Reconocer la observación, el análisis, la } \\
\text { planeación y la acción proponiendo un proce- } \\
\text { so humano de mejora permanente. La gestión } \\
\text { de cambio es una intervención en la cultura } \\
\text { institucional, en el comportamiento del líder } \\
\text { y depende de un ejercicio de liderazgo con } \\
\text { capacidad para inspirar y transformar. }\end{array}$ \\
\hline
\end{tabular}

enmarcan la estructura jerárquica de las instituciones y tiene, en consecuencia, unas escalas disímiles en cada país. Las posibles diferencias, incluso abismales, entre unos sujetos autodeterminados y otros, se reducen en la medida que estilos como el participativo-democrático consolidan la confianza profesional en el líder, de modo que aumentan en el sujeto la credibilidad y la confianza entre las personas que actúan con responsabilidad y rectitud.

Vinculado a lo anterior, se puede concluir que el impacto de los atributos del líder policial depende del grado de autonomía de quien lo ejerce. Estas características que la teoría sustenta (Nieto et al., 20I8) pueden estandarizarse $y$, bajo ejercicios de resolución de conflictos y toma de decisiones, favorecen el flujo de trabajo y la creación de condiciones propias para responder eficazmente a las demandas del servicio policial en cualquier contexto sociocultural.

La necesidad de capacitación en liderazgo policial para la Rinep ha de responder, a su vez, al llamado por un 
liderazgo transformacional, posiblemente tomando como base la teoría de Bass (1985), la cual, en diálogo con un modelo integrador, favorezca - como ya lo anticiparon Espinoza-Parra et al. (2015) - el trabajo sinérgico que en el líder ejerce una función de mediador y así alcanzar la satisfacción en el contexto institucional con mayor eficacia al lograr el compromiso de todos los miembros de las unidades lideradas.

Dadas las diferencias y similitudes señaladas, se denota la necesidad formativa que Day (20I I como se cita en Flynn \& Herrington, 20I5), señalaba en cuanto a las condiciones disímiles que implica desarrollar el liderazgo y entrenar en el liderazgo. Teniendo en cuenta que no basta con los conocimientos que el líder tiene sobre las situaciones del contexto que lo rodean, se deben desarrollar unas capacidades suficientes para que el líder policial ejerza con compromiso la gestión, teniendo en cuenta unos indicadores pertinentes, tal como lo señala Rangel (2015), según la normatividad de la institución y el país. Este tipo de formación ha de considerar los diferentes niveles de responsabilidad del líder policial, sus capacidades emocionales, cognitivas y físicas, así como un estilo propio de líder policial según las características de su contexto cultural y social.

Cabe señalar que, de acuerdo con UNODC (20II), existe una estrecha relación entre el liderazgo, la cultura y la conducta, lo cual conduce a una mirada prospectiva en las que el atributo, la competencia, las características propias de los procesos comunicativos y las necesidades de socializar las buenas prácticas en diversas latitudes implica para esta propuesta un reto aún mayor en el que converjan la formación teórica, el desarrollo del liderazgo y los procesos comunicacionales que se pongan al servicio del ejercicio de liderazgo, aprovechando las facilidades de acceso a las herramientas tecnológicas que permiten crear e incursionar en innovaciones imposibles hace pocos años.

\section{Conclusiones}

En este apartado se da cuenta de tres escenarios a los que conduce la investigación adelantada en conjunto con la participación de nueve cuerpos de policía miembros de la Rinep. Estos escenarios se describen en términos del cumplimiento de los objetivos propuestos, la relevancia del aparato metodológico aplicado y la prospectiva de los resultados mismos de la investigación.
En primer lugar, la propuesta de liderazgo policial que responde a las perspectivas de los países participantes se sustenta en el análisis de particularidades y estilos propios de cada contexto. Vale la pena recalcar que no es pretensión de esta propuesta homogeneizar las condiciones de ser líder policial, sino plantear una base suficiente, sólida y pertinente que favorezca el desarrollo de liderazgos capaces de responder a las exigencias de la realidad. Los componentes de esta propuesta aportan, a su vez, a varias líneas de acción propias de la Rinep, de modo que se ha cumplido el propósito de contribuir a las estrategias de cooperación e integración de los cuerpos de policía miembros de la red.

En segundo lugar, la investigación permitió identificar, mediante una metodología de estudio comparativo, no solo las particularidades de cada institución, sino los aspectos similares y disímiles a partir de los cuales esta propuesta de liderazgo policial entra en discusión con las miradas propias de cada contexto $y$, a su vez, se enriquece con la trayectoria de cada apuesta local. De este modo, el Diplomado Internacional en Liderazgo Policial, construido con elementos de los programas ya existentes en otras regiones para la profesionalización policial, se considera una propuesta formativa que se sirve de los recursos tecnológicos más utilizados, lo que desde el estudio comparado se proyecta como indicadores de éxito en su implementación.

El tercer lugar al que conduce la investigación se proyecta en el escenario futuro, así como propone el compromiso y la corresponsabilidad de los miembros de la Rinep. Los procesos formativos tienen la doble entrada de quienes imparten una enseñanza y quienes la reciben, y para este caso se agrega una entrada adicional: la posibilidad de hacer que la gestión del conocimiento sea permanente, alcanzando así el propósito del trabajo en red al promover de forma permanente estrategias y acciones que favorezcan a todos los miembros.

Uno de los compromisos inmediatos se cifra en establecer el diálogo indispensable para validar y avalar el diplomado internacional. Al estar dirigido a los encargados de la toma de decisiones institucionales, este proceso formativo reviste una gran relevancia y se cualificará con los aportes de las distintas instancias de la red, con las cuales se realizará el trabajo colaborativo para su implementación.

\section{Conflicto de intereses}

El artículo es resultado de la investigación "Liderazgo policial: un aporte a las estrategias de cooperación e 
integración de cuerpos de policía miembros de Rinep". La Red de Internacionalización Educativa Policial (Rinep) son cuerpos de policía e instituciones universitarias vinculadas al proyecto: Argentina (Policía de la Provincia de Jujuy), Colombia (Dirección Nacional de EscuelasEscuela de Posgrados de Policía), Chile (Escuela de Investigaciones de Chile Escipol y Policía de Investigaciones), El Salvador (Academia Nacional de Seguridad), Honduras (Policía Nacional y Universidad Nacional de la Policía de Honduras), México (Universidad de Ciencias de la Seguridad Nuevo León y el Instituto Estatal de Estudios Superiores en Seguridad y Profesionalización Policial del Estado de Michoacán), Panamá (Servicio Nacional de Fronteras), Perú (Policía Nacional) y República Dominicana (Policía Nacional).

\section{Referencias}

Avolio, B., \& Bass, B. (2004). Multifactor leadership questionnaire. Mind Garden.

Bardin, L. (2002). Análisis de contenido ( $3^{\mathrm{a}}$ ed.). Akal Ediciones.

Bass, B. (1985). Leadership and performance beyond expectations. The Free Press.

Bass, B. M., \& Avolio, B. (1993). Transformational leadership and organizational culture. Public Administration Quarterly, I7(I), II2-12I.

Bennis, W., \& Nanus, B. (1985). Leaders: the strategies for taking charge. Harper \& Row.

Cisterna, F. (2005). Categorización y triangulación como procesos de validación del conocimiento en investigación cualitativa. Theoria, I4(I), 6I-7I.

Espinoza-Parra, S., Molero, F., \& Fuster-Ruiz de Apodaca, M. J. (2015).Transformational leadership and job satisfaction of police officers (carabineros) in Chile: the mediating effects of group identification and work engagement. Revista de Psicologia Social, 30(3), 439-467.

Flynn, E. A., \& Herrington,V. (2015). Toward a profession of police leadership. New perspectives in policing bulletin. U.S. Department of Justice, National Institute of Justice. https:// www.ncjrs.gov/pdffiles I/nij/248573.pdf

Goodrick, D. (2014). Estudios de caso comparativos, sintesis metodológicas: evaluación de impacto $N .^{\circ} 9$. Centro de Investigaciones de Unicef. https://www.unicef-irc.org/publications/ pdf/MB9ES.pdf
Hernández, R., Fernández, C., \& Baptista, P. (20I4). Metodología de la investigación ( $6^{\mathrm{a}}$ ed.) McGraw-Hill.

Hersey, P., \& Blanchard, K. H. (1967). Liderazgo situacional. http://www.metamanagers.com/documents/Liderazgo_ Situacional.pdf

Hoggett, J., Redford, P., Toher, D., \& White, P. (20I8) Challenges for police leadership: identity, experience, legitimcy and direct entry.

Lewin, K., Lippitt, R., \&White, R. (1939). Patterns of aggressive behavior in experimentally created "social climates". The Journal of Social Psychology, 10(2), 269-308. https://doi.org/ I0.1080/00224545.1939.97|3366

Nieto, J. H., Nieto, J. C., \& Moreno, J. (2018). Modelo holístico de liderazgo policial. Revista Logos Ciencia \& Tecnología, I0(2), 90-I I0. https://doi.org/ |0.22335/rlct.v I 0i2.55 |

OCDE (Organización para la Cooperación y Desarrollo Económico). (2008). Education at a glance. OECD Indicators.

Paz,A. (2009). El policía, líder de la comunidad para el logro de la convivencia y seguridad ciudadana. Universidad de la Sabana.

Pedraza, L., Villamizar, A., \& Ortíz, Z. (20I5). Desafíos del proceso de construcción de paz para la Policía Nacional de Colombia. Revista Criminalidad, 57(3), I35-I 48.

Rangel, P. R. (20I5). Liderazgo policial. Para la formación del funcionario del siglo XXI. https://latinamerica.hss.de/filead$\mathrm{min} /$ user_upload/Projects_HSS/Latin_America/Migration-230607/Liderazgo_Policial.pdf

Reddin, W. J. (1983). Management effectiveness and style individual or situation. University of New Brunswick.

Sánchez, J. F. (2010). Liderazgo: teorías y aplicaciones. Universidad Pontificia de Salamanca.

Unesco (Organización de las Naciones Unidas para la Educación, la Ciencia y la Cultura), \& OIE (Oficina Internacional de Educación). (1998). Conferencia Mundial sobre la Educación Superior. La educación superior en el siglo XXI. Tomo I-Informe final.

UNODC (United Nations Office on Drugs and Crime). (20II). Handbook on police accountability oversight and integrity. Criminal Justice Handbook Series. https://www. unodc.org/pdf/criminal_justice/Handbook_on_police_ Accountability_Oversight_and_Integrity.pdf

Yukl, G., \& Falbe, C. M. (199I). The importance of different power sources in downward and lateral relations. Journal of Applied Psychology, 76, 416-423. 
Yukl, G., \& Fleet, D. D. van. (1992). Theory and research in organizations. En M. D. Dunnette, \& L. M. Hough (Eds.), Handbook of industrial and organizational psychology ( $2^{\mathrm{a}}$ ed., Vol. 3, pp. 147-197). Consulting Psychologists Press.

Zanini, M., Conceicao, M., \& Migueles, C.(2018). Uma análise dos antecedentes da confiança no líder numa unidade policial de operações especiais. Revista de Administración Pública, 52(3). 45 I-468. https://doi.org/ | 0. I590/0034-76 I 2 I678 I I
Zanini, M., Migueles, C., \& Colmerauer, M. (20|4). A ponta da lança. Editora Elsevier.

Zaleznik, A. (1977). Managers and leaders: are they different? Harvard Business Review, 55(3), 67-78. 